\title{
Multimodal effective connectivity analysis reveals seizure focus and propagation in musicogenic epilepsy
}

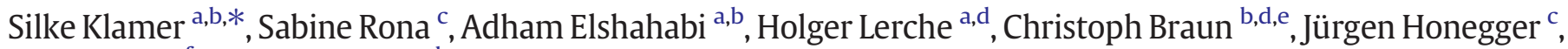 \\ Michael Erb ${ }^{\mathrm{f}}$, Niels K. Focke ${ }^{\mathrm{a}, \mathrm{d}}$ \\ a Department of Neurology and Epileptology, Hertie-Institute for Clinical Brain Research, University of Tuebingen, Tuebingen, Germany \\ ${ }^{\mathrm{b}}$ MEG Center, University of Tuebingen, Tuebingen, Germany \\ c Department of Neurosurgery, University of Tuebingen, Tuebingen, Germany \\ d Werner Reichardt Centre for Integrative Neuroscience, Tuebingen, Germany \\ e CIMeC, Center for Mind/Brain Sciences, University of Trento, Trento, Italy \\ ${ }^{\mathrm{f}}$ Department of Biomedical Magnetic Resonance, University of Tuebingen, Tuebingen, Germany
}

\section{A R T I C L E I N F O}

\section{Article history:}

Accepted 12 March 2015

Available online 20 March 2015

\section{Keywords:}

fMRI

High-density EEG

MEG

Musicogenic epilepsy

Effective connectivity

Dynamic causal modeling

\begin{abstract}
A B S T R A C T
Dynamic causal modeling (DCM) is a method to non-invasively assess effective connectivity between brain regions. 'Musicogenic epilepsy' is a rare reflex epilepsy syndrome in which seizures can be elicited by musical stimuli and thus represents a unique possibility to investigate complex human brain networks and test connectivity analysis tools. We investigated effective connectivity in a case of musicogenic epilepsy using DCM for fMRI, high-density (hd-) EEG and MEG and validated results with intracranial EEG recordings.

A patient with musicogenic seizures was examined using hd-EEG/fMRI and simultaneous '256-channel hd-EEG'/ 'whole head MEG' to characterize the epileptogenic focus and propagation effects using source analysis techniques and DCM. Results were validated with invasive EEG recordings.

We recorded one seizure with hd-EEG/fMRI and four auras with hd-EEG/MEG. During the seizures, increases of activity could be observed in the right mesial temporal region as well as bilateral mesial frontal regions. Effective connectivity analysis of fMRI and hd-EEG/MEG indicated that right mesial temporal neuronal activity drives changes in the frontal areas consistently in all three modalities, which was confirmed by the results of invasive EEG recordings. Seizures thus seem to originate in the right mesial temporal lobe and propagate to mesial frontal regions.

Using DCM for fMRI, hd-EEG and MEG we were able to correctly localize focus and propagation of epileptic activity and thereby characterize the underlying epileptic network in a patient with musicogenic epilepsy. The concordance between all three functional modalities validated by invasive monitoring is noteworthy, both for epileptic activity spread as well as for effective connectivity analysis in general.
\end{abstract}

(c) 2015 Elsevier Inc. All rights reserved.

\section{Introduction}

Many physiological and pathological processes of the human brain are driven by networks and connectivity between brain regions. Thus, analysis of these connections is of particular importance in neurosciences and several methods have been proposed to study connectivity in-vivo by means of functional imaging. One of these is dynamic causal modeling (DCM), a method to assess the effective connectivity between brain regions, i.e. the causal influence that one neuronal system exerts over others. It was first developed for fMRI with the aim to estimate the parameters of a neuronal system model from which a blood

\footnotetext{
* Corresponding author at: Department of Neurology and Epileptology, Hertie-Institute for Clinical Brain Research, University of Tuebingen, Hoppe-Seyler-Strasse 3, 72076 Tuebingen, Germany. Fax: + 497071294488.

E-mail address: silke.klamer@uni-tuebingen.de (S. Klamer).
}

oxygenation level dependent (BOLD) signal can be predicted that corresponds as closely as possible to the measured BOLD time series (Friston et al., 2003). Later, DCM was extended to the analysis of effective connectivity in EEG and MEG data taking into account the very high temporal resolution of these techniques and thereby combining a spatial forward model with a biologically informed temporal forward model (Kiebel et al., 2008). DCM can be used to test which brain region drives which by constructing different models of interacting regions or nodes and identifying the best one by model comparison. This is especially interesting in epilepsy where the identification of the epileptic focus, i.e. the brain region - or node - driving the others during epileptic discharges, and the propagation of epileptic activity is not only of clinical interest but could also serve as a model condition for the investigation of brain connectivity in general.

'Musicogenic epilepsy' is a rare reflex epilepsy syndrome, in which seizures are triggered by musical stimuli. This condition gives the 
unique possibility to specifically evoke seizures, i.e. functional changes in complex brain networks in humans, non-invasively. Seizure origin has been shown to involve foci within the temporal lobes with a rightsided preponderance in most cases, but extra-temporal foci have also been described (for review see Pittau et al., 2008). Several (EEG-) fMRI-studies have even postulated that stimulus-induced activity in frontal cortical areas, which are believed to be key structures in processing emotional aspects of music, may initiate the seizure cascade in temporal regions finally visible as epileptic discharges in scalp EEG (Marrosu et al., 2009; Morocz et al., 2003; Pittau et al., 2008).

We investigated the epileptic networks in a case of musicogenic epilepsy, in which we were able to specifically elicit seizures, using dynamic causal modeling (DCM) for high-density EEG (hd-EEG), MEG and fMRI to identify the seizure onset zone and the further temporal sequence of epileptic activity spread and to investigate whether early frontal activations precede seizure activity in temporal regions or if the epileptogenic focus is located within the temporal lobe. We were able to validate our results with intracranial EEG recordings.

\section{Methods}

Subject

A 22-year-old right-handed man suffered from focal epilepsy with déjà-vu auras, dyscognitive and secondarily generalized tonic-clonic seizures since six years of age. Seizures could be provoked by rap music (no other types of music), but also occurred spontaneously with increasing frequency. Under a treatment with lamotrigine $600 \mathrm{mg} /$ day, levetiracetam $1000 \mathrm{mg} /$ day and lacosamide $300 \mathrm{mg} /$ day he still experienced 2-5 (spontaneous or evoked) seizures per month, often in clusters. Conventional scalp video-EEG showed a right temporal origin with a typical anterior temporal spike at the beginning of all provoked and spontaneous seizures. This initial spike was followed by a seizure pattern of rhythmic delta activity ( 2 Hz) (see Fig. 1). Structural MRI showed no lesion on visual expert reading, language fMRI left-hemispheric language dominance and neuropsychological testing revealed deficits in non-verbal memory performance indicating right mesial temporal dysfunction.

\section{Multimodal imaging study - data acquisition}

After giving his informed consent, the patient underwent simultaneous hd-EEG-fMRI as well as simultaneous hd-EEG and MEG in order to record seizures triggered by rap music.

\section{Simultaneous hd-EEG-fMRI recording}

Hd-EEG/fMRI was recorded using a 256-channel EEG system (Electrical Geodesics, Inc., Eugene, OR, U.S.A.) within a 3 T Scanner (Siemens MAGNETOM Trio, Siemens AG, Erlangen, Germany). We acquired a sagittal T1-weighted 3D-MPRAGE sequence as high-resolution anatomical reference (TR $2.3 \mathrm{~s}$, TE $3.03 \mathrm{~ms}$, FA $8^{\circ}$, voxel size $1 \times 1 \times 1 \mathrm{~mm}$ ); a field map was also recorded for later correction of distortions in the functional images caused by magnetic field inhomogeneity. For the functional sequence gradient-echo planar $\mathrm{T} 2 *$-weighted images covering the whole brain (TR 2 s, TE $32 \mathrm{~ms}$, FA $90^{\circ}$, voxel size $3 \times 3 \times 4 \mathrm{~mm}$ ) with simultaneous hd-EEG were acquired. The sequence consisted of five minutes of rest, followed by two minutes of nonepileptogenic music (Bryan Adams, "Everything I Do"), another five
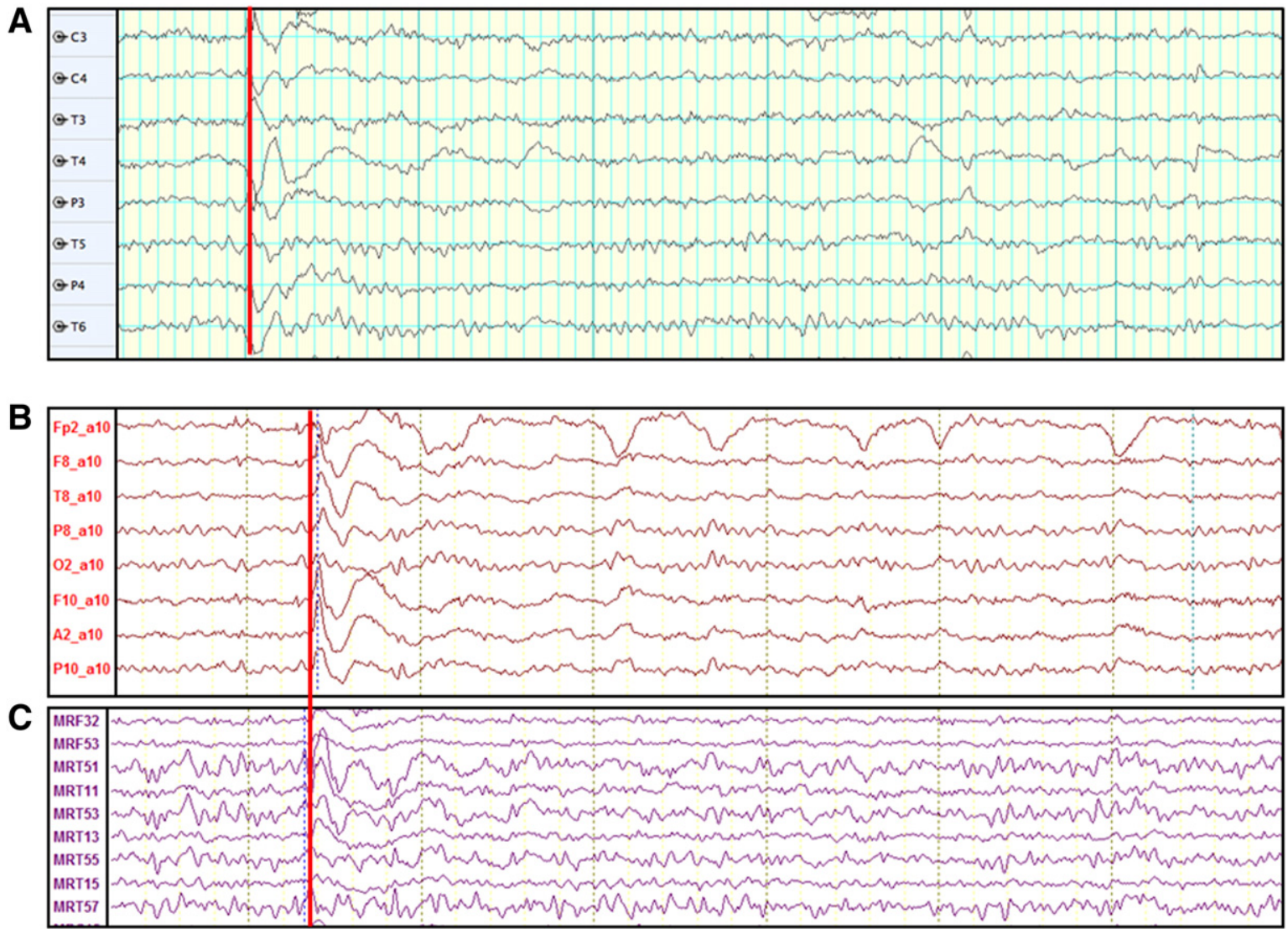

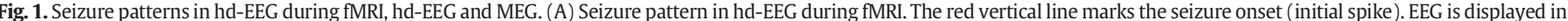

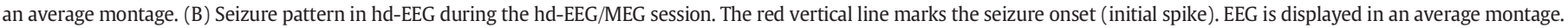

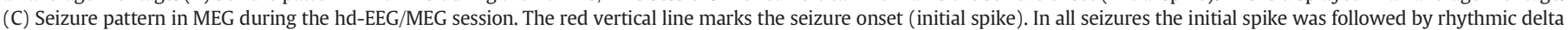
activity $(\sim 2 \mathrm{~Hz})$. 
minutes of rest and then two pieces of epileptogenic rap music the patient knew to elicit seizures reliably (Eminem, "To Life" and "Lose Yourself") played in a loop for $3.5 \mathrm{~min}$, until the appearance of a seizure which the patient indicated by button press.

\section{Simultaneous hd-EEG and MEG recording}

Simultaneous hd-EEG/MEG recording was performed using a 275channel whole-head MEG-system (VSM MedTech Ltd., Coquitlam, Canada) and the same 256-channel hd-EEG within a shielded room. The patient was lying on his back in a supine position and was asked to keep his eyes closed during the measurement. To reduce head movements, his head was stabilized with cushions. The sampling rate in MEG was $3906.3 \mathrm{~Hz}$ and in hd-EEG $1000 \mathrm{~Hz}$. The design was similar as in the hd-EEG/fMRI measurement: 5 min rest - 1 min non-epileptogenic music - 5 min rest -6 min epileptogenic music until the recording of seizures.

\section{Multimodal imaging study - data analysis}

\section{Whole-brain mapping of music related BOLD changes}

For the fMRI analysis, hd-EEG-data were corrected for MR- and cardioballistic artifacts, the onset of the seizure was marked by an experienced EEG reader upon visual inspection (see Fig. 1 for illustration of the seizure) and the exact time point was used for further analysis of the fMRI data using SPM (SPM8, v5236 Wellcome Trust Centre for Imaging Neuroscience; http://www.fil.ion.ucl.ac.uk/spm). In a first step, functional images were checked manually for head motion and the last 18 scans were discarded due to movement artifacts in the course of the seizure. The remaining 451 functional scans underwent slice timing procedure, were realigned and unwarped based on the estimated field map data, co-registered to the anatomical reference image, normalized to MNI space and smoothed with an isotropic Gaussian kernel ( $8 \mathrm{~mm}$ FWHM). A quality check of the realignment parameters revealed that head movement remained $<5 \mathrm{~mm}$ throughout the whole session. Spatial normalization was performed to be able to compare source analysis results and calculate Euclidean distances between modalities, especially with MEG whose analysis is not based on the individual but on a standard spherical head model. The blocks of non-epileptogenic music, epileptogenic music and seizure were then convolved with the canonical hemodynamic response function in order to obtain main effects for (i) non-epileptogenic music vs. rest and (ii) seizure vs. epileptogenic music. We did not model the main effect for epileptogenic music vs. rest and vs. non-epileptogenic music as that block with its $152 \mathrm{~s}$ until the beginning of the seizure was rather long and therefore contained too much low-frequency noise to obtain a valid contrast.

\section{Hd-EEG and MEG source analysis}

Hd-EEG and MEG data were also visually inspected and seizure initiating spikes were identified and marked by an experienced EEG reader (see Fig. 1 for illustration of one exemplary seizure). Source analysis of the onset of each seizure as well as the average of all seizure initiating spikes in MEG was performed using BESA Research 5.3.7 (Brain Electrical Source Analysis, BESA GmbH, Gräfelfing, Germany) and a spherical head model. Hd-EEG data was analyzed using the Cartool software (brainmapping.unige.ch/ cartool) and a simplified realistic head model called LSMAC (Locally Spherical Model with Anatomical Constraints) derived from the individual MRI, as we were able to show in a previous study that the use of individual realistic head models improves EEG source analysis results (Klamer et al., 2014). For both data sets a distributed source model (LORETA, low resolution brain electromagnetic tomography) was applied. To investigate possible propagation effects, source analysis was not only performed at the mid-point of the ascending phase as suggested by Lantz et al. (2003), but also at the onset and the maximum negativity of the initial spike. Talairach coordinates of the center-of-mass of the source analysis results were determined and converted to MNI space (Montreal Neurologic Institute Atlas, MNI)
(Mazziotta et al., 1995) using Brett's function (http://imaging.mrc-cbu. cam.ac.uk/imaging/MniTalairach). Euclidean distances between the center-of-mass of the hd-EEG, MEG and fMRI sources were calculated to determine localization accuracy.

Furthermore, we analyzed power spectra at the onset of each seizure (seizure initiating spike) and throughout the whole seizure after correction of the data for eye movement artifacts.

\section{Effective connectivity analyses}

Based on the results of the fMRI analysis, i.e. the seizure-related BOLD map, and previous literature (Marrosu et al., 2009; Morocz et al., 2003; Pittau et al., 2008; Tayah et al., 2006; Trevathan et al., 1999) two regions of interest (ROI) were selected as possible seizure origins in this case: the frontal (MNI: $260-14 \mathrm{~mm}$ ) and the right mesiotemporal (MNI: $386-12 \mathrm{~mm}$ ) cluster of the fMRI activation map. To perform the effective connectivity analysis based on fMRI we computed the first principal eigenvariate of the voxel time series for each ROI and mean-corrected it using the "effects of interest" F-contrast. For the hdEEG and MEG effective connectivity analysis we averaged the seizure initiating spikes of all recorded seizures and chose the time from the onset of the spike until the maximum negativity for further analysis. Using dynamic causal modeling (DCM) in SPM8 we constructed 2 models, each with one ROI acting as autonomous input over the other one with a modulatory effect of seizure on the connections of the two ROIs (Fig. 2A). After estimation of the parameters of each model within each modality they were compared using Bayesian model comparison, in which selection of the most appropriate model is made using the difference in their log-evidence scores (Kiebel et al., 2008).

\section{Intracranial EEG recordings}

To determine the seizure origin and thus the epileptic focus in this patient, intracranial EEG recordings were performed. Two depth electrodes were implanted in the perisylvian region, one in the right mesial frontal region. Further, subdural strip electrodes were implanted covering the lateral as well as basal aspects of the right temporal lobe including the hippocampus. Invasive video-EEG monitoring was performed and the seizure onset zone was determined by an experienced epileptologist.

\section{Results}

\section{Hd-EEG/fMRI analysis}

During the hd-EEG/fMRI session a seizure occurred after $152 \mathrm{~s}$ of listening to epileptogenic rap music and lasted for $25 \mathrm{~s}$ until the session had to be aborted due to movement. There were no interictal epileptic discharges in this session. Having experienced one seizure inside the scanner the patient felt very exhausted and did not consent to another provocation inside the scanner.

Listening to non-epileptogenic music vs. rest provoked BOLD signal increases in the right temporal lobe, in a region corresponding to the primary auditory cortex, as well as left temporal, occipito-parietal and cerebellar areas ( $p<0.05$, FWE corrected) (Supp. Fig. 1).

During the seizure (vs. epileptogenic music) the BOLD global maximum was located in the right mesial temporal lobe, more precisely in the right hippocampus extending to the right insula and the right amygdala. Further, we observed activations in the right posterior middle temporal gyrus, right thalamus, bilateral medial frontal lobe and the right precentral gyrus (patient indicated seizure by button press with the left thumb) ( $p<0.05$, FWE corrected) (Fig. 2). For MNI coordinates of activations see Table 1 .

\section{Hd-EEG and MEG source analysis}

In the hd-EEG/MEG session we were able to record four auras. All were evoked by the epileptogenic music and were characterized by a 


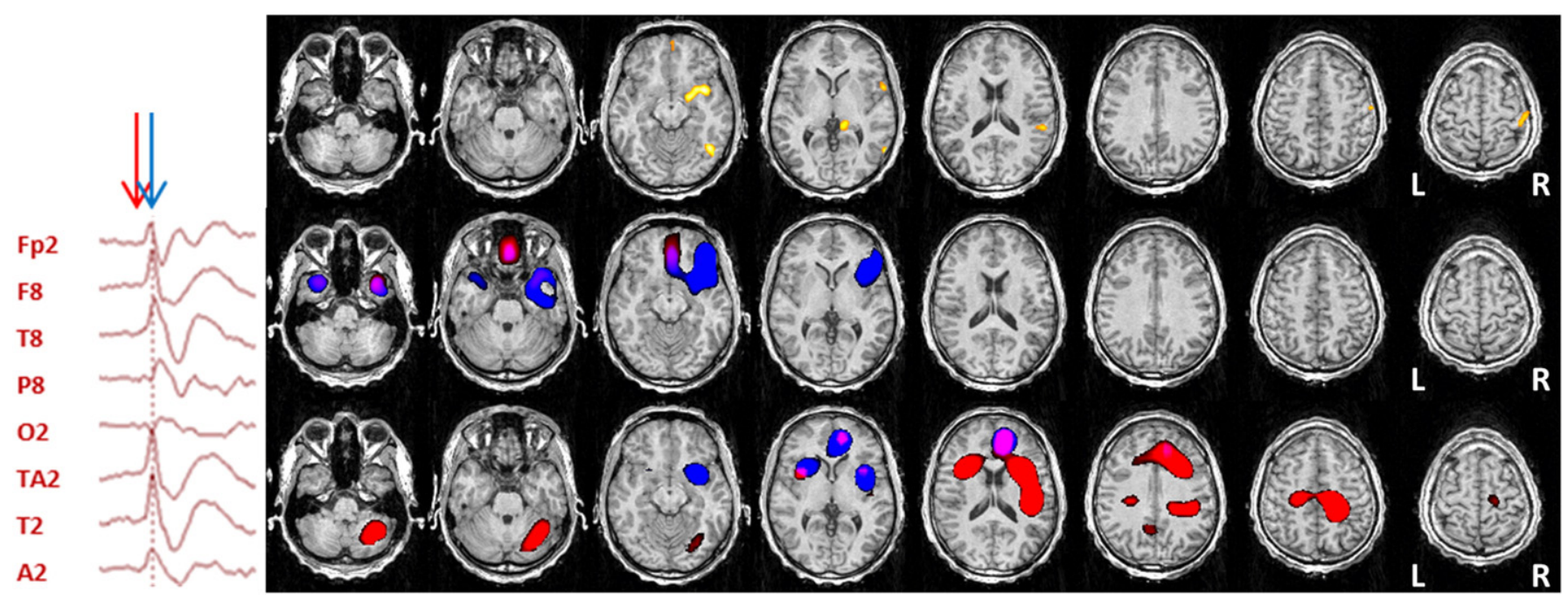

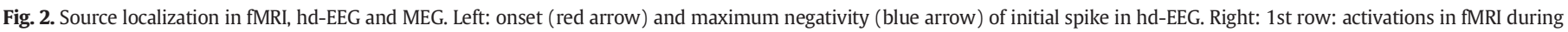

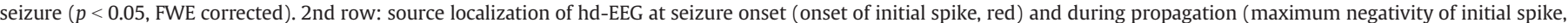

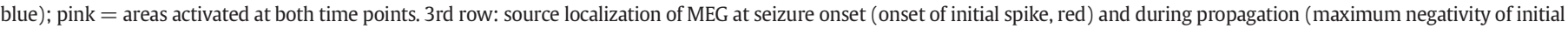
spike, blue); pink = areas activated at both time points.

déjà-vu feeling. The duration ranged from 5.0 s to 19.7 s (mean $10.53 \pm$ $6.71 \mathrm{~s})$.

Source analysis of hd-EEG data showed sources in the bilateral mesial frontal and the right as well as the left mesial temporal regions at seizure onset (onset of initial spike) as well as at the mid-point of the ascending phase of the initial spike. During further propagation, i.e. at the maximum negativity of the initial spike, frontal and mesial temporal sources remained stable and an additional source in the right basal frontal region evolved (Fig. 2). MNI coordinates of the sources are listed in Table 1.

Analysis of seizure onset using MEG data also revealed sources in the right mesial frontal and bilateral mesial temporal regions, but also in the right posterior temporal lobe, parietal and cerebellar areas. At the midpoint of the ascending phase of the initial spike and during propagation, the sources in the right posterior temporal lobe and the parietal and cerebellar areas disappeared while the other sources remained (Fig. 2). For MNI coordinates of all sources see Table 1 .

Euclidean distances between hd-EEG, MEG and fMRI were calculated for the centers-of-mass of the frontal and the right mesial temporal sources during onset and propagation of the initial spike and the results are listed in Table 2. Euclidean distances ranged from 13 to $32 \mathrm{~mm}$ for the frontal source and from 9 to $52 \mathrm{~mm}$ for the right mesio-temporal source. While hd-EEG sources and consequently distances to the other modalities remained stable for all three time points, MEG sources at the onset of the initial spike showed the largest distances to the other modalities, especially for the right mesio-temporal source. MEG sources at the mid-point of the ascending phase of the initial spike were located closest to hd-EEG and fMRI.

Analysis of power spectra of hd-EEG data showed a peak at $3 \mathrm{~Hz}$ in the right temporal region at seizure onset. Throughout the whole seizure a peak at $1.9 \mathrm{~Hz}$ was visible in the right temporal region corresponding to the visually identified seizure pattern (Supp. Fig. 2).

\section{Effective connectivity analyses}

Bayesian model comparison identified model 2 to be significantly better than model 1, i.e. neuronal activity originating in the right mesial temporal region drives the frontal ROI (Fig. 3). It is of particular note that these findings were consistent between effective connectivity analyses of all three modalities.

\section{Results of intracranial EEG recordings}

During invasive video-EEG monitoring nine spontaneous and evoked seizures were recorded. Seizure origin was identified in all seizures at the contacts of the strip electrode localized below the right hippocampus with fast propagation to the mesial frontal depth electrode within 100-150 ms (Fig. 4). Interictal epileptic discharges were recorded mostly at the site of the seizure origin, i.e. the strip electrode below the right hippocampus, but isolated spikes at the mesial frontal depth electrode could be observed as well.

Based on these results, epilepsy surgery was performed with right amygdalo-hippocampectomy and resection of the right temporal pole. Histological examination of the resected tissue showed a low-grade hippocampal sclerosis. Postoperatively (six months follow up) the patient experienced just a single, provoked seizure which is a dramatic reduction of seizure frequency and indicates successful surgery.

Table 1

MNI coordinates ( $\mathrm{mm}$ ) of sources identified by hd-EEG, MEG and fMRI

\begin{tabular}{|c|c|c|c|c|c|c|c|}
\hline Brain region & $\begin{array}{l}\text { Onset hd-EEG } \\
(\mathrm{xyz})\end{array}$ & $\begin{array}{l}\text { Mid-point } \\
\text { hd-EEG (xyz) }\end{array}$ & $\begin{array}{l}\text { Maximum negativity } \\
\text { hd-EEG (xyz) }\end{array}$ & $\begin{array}{l}\text { Onset MEG } \\
(\mathrm{xyz})\end{array}$ & $\begin{array}{l}\text { Mid-point } \\
\text { MEG (xyz) }\end{array}$ & $\begin{array}{l}\text { Maximum negativity } \\
\text { MEG (xyz) }\end{array}$ & $\begin{array}{l}\text { fMRI } \\
(\mathrm{xyz})\end{array}$ \\
\hline Right frontal & $2.1437 .95-15.53$ & 2.14 37.95-15.53 & $2.1437 .95-15.53$ & 10.6146 .9713 .03 & 10.6146 .9713 .03 & $3.5440 .48-2.97$ & $260-14$ \\
\hline Right temporo-mesial & $23.6116 .73-31.96$ & $23.6116 .73-31.96$ & $23.6112 .36-32.21$ & 31.8210 .5718 .73 & $38.8911 .65-4.45$ & $38.89-2.76-5.29$ & $386-12$ \\
\hline Right fronto-basal & $\mathrm{N} / \mathrm{A}$ & $\mathrm{N} / \mathrm{A}$ & $27.9033 .36-10.73$ & $\mathrm{~N} / \mathrm{A}$ & $\mathrm{N} / \mathrm{A}$ & $\mathrm{N} / \mathrm{A}$ & $\mathrm{N} / \mathrm{A}$ \\
\hline Left temporal & $-23.6116 .73-31.96$ & $-23.6116 .73-31.96$ & $-23.6116 .73-31.96$ & -31.8210 .9311 .13 & -31.8211 .293 .53 & -24.7518 .53 .91 & $\mathrm{~N} / \mathrm{A}$ \\
\hline Right posterior-temporal & $\mathrm{N} / \mathrm{A}$ & $\mathrm{N} / \mathrm{A}$ & $\mathrm{N} / \mathrm{A}$ & $45.96-25.4716 .83$ & $\mathrm{~N} / \mathrm{A}$ & $\mathrm{N} / \mathrm{A}$ & $44-58-15$ \\
\hline
\end{tabular}


Table 2

Euclidean distances $(\mathrm{mm}$ ) between sources identified by hd-EEG, MEG and fMRI.

\begin{tabular}{|c|c|c|c|c|c|c|c|c|c|}
\hline Brain region & $\begin{array}{l}\text { ED onset } \\
\text { hd-EEG-fMRI }\end{array}$ & $\begin{array}{l}\text { ED Mid-point } \\
\text { hd-EEG-fMRI }\end{array}$ & $\begin{array}{l}\text { ED maximum } \\
\text { negativity } \\
\text { hd-EEG-fMRI }\end{array}$ & $\begin{array}{l}\text { ED onset } \\
\text { MEG-fMRI }\end{array}$ & $\begin{array}{l}\text { ED } \\
\text { mid-point } \\
\text { MEG-fMRI }\end{array}$ & $\begin{array}{l}\text { ED maximum } \\
\text { Negativity } \\
\text { MEG-fMRI }\end{array}$ & $\begin{array}{l}\text { ED onset } \\
\text { hd-EEG-MEG }\end{array}$ & $\begin{array}{l}\text { ED mid-point } \\
\text { hd-EEG-MEG }\end{array}$ & $\begin{array}{l}\text { ED maximum } \\
\text { negativity } \\
\text { hd-EEG-MEG }\end{array}$ \\
\hline Right frontal & 22.1 & 22.1 & 22.1 & 31.21 & 31.21 & 22.47 & 31.13 & 31.13 & 13.06 \\
\hline Right temporo-mesial & 26.84 & 26.84 & 25.61 & 31.68 & 9.47 & 11.07 & 51.72 & 31.88 & 34.45 \\
\hline
\end{tabular}

\section{Discussion}

Musicogenic seizures are a rare opportunity to study pathological neuronal activity in-vivo in a controlled fashion enabling unique insights into brain functions. Here we investigated epileptic networks in a patient with musicogenic seizures triggered by rap music using DCM in a multimodal approach consisting of hd-EEG/fMRI and simultaneous hd-EEG and MEG. While each of these methods alone has been shown to be able to localize epileptic activity (Brodbeck et al., 2011; Pataraia et al., 2005; Vulliemoz et al., 2009), we made use of their complementary nature to improve analyses with regards to localization accuracy and effective connectivity and assess if the modalities will give concordant results. fMRI is able to show hemodynamic changes in the whole brain time-locked to epileptic activity and is characterized by its very high spatial resolution, especially for sources deep in the brain. The advantage of EEG and MEG compared to fMRI is their very high temporal resolution in the range of milliseconds.

Consistent across all three modalities we were able to observe activations in the mesial frontal and right mesial temporal regions at seizure onset. Euclidean distances between modalities indicated good concordance between modalities and were within the methodological limits described in our previous study (Klamer et al., 2014).

This is reassuring and highlights the ability of our chosen multimodal technique to correctly localize the epileptogenic focus, especially in combination with the findings of video-EEG, neuropsychological testing and especially the results of the invasive EEG recordings, that all indicated a right mesial temporal focus. Further, histological examination after amygdalo-hippocampectomy showed a hippocampal sclerosis, which is a common cause for epilepsy in adults. Certain existing discrepancies between source analysis results, in particular between hd-EEG and MEG, may be attributed to the fact that EEG is dominated by radial sources, whereas MEG detects only tangential sources. Therefore, the combination of both methods should give a more complete picture of the underlying epileptic activity and yield information not available from either technique alone (Barkley and Baumgartner, 2003).

\section{Dynamic causal modeling}

Using dynamic causal modeling, we were able to investigate the epileptic network in this patient and thereby to determine the seizure onset zone and the further temporal sequence of epileptic activity spread. Effective connectivity analysis of fMRI as well as hd-EEG and MEG concordantly revealed that seizure activity was driven by the right mesial temporal region, the observed co-activation in the mesial frontal lobes appeared secondary. This was confirmed by the results of the intracranial EEG recordings, which clearly located the seizure onset zone in the right hippocampus, a structure crucial for nonverbal memory processes (Kelley et al., 1998; Smith and Milner, 1981), and revealed propagation of epileptic activity to the mesial frontal lobes with a short temporal delay of 100 to 150 ms (Fig. 4).

Therefore, we were not able to confirm previously published hypotheses about an initial frontal cortical involvement triggering the
A

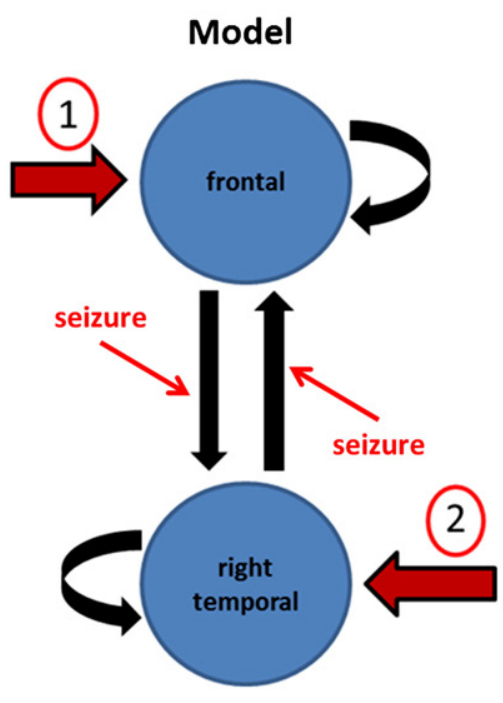

B
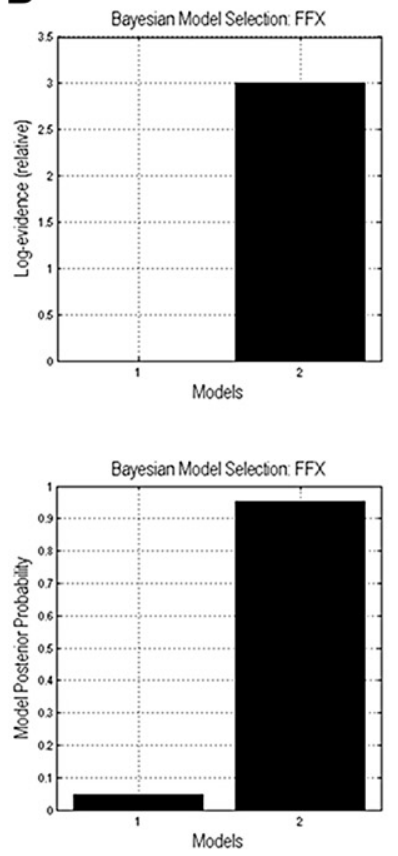

fMRI
C
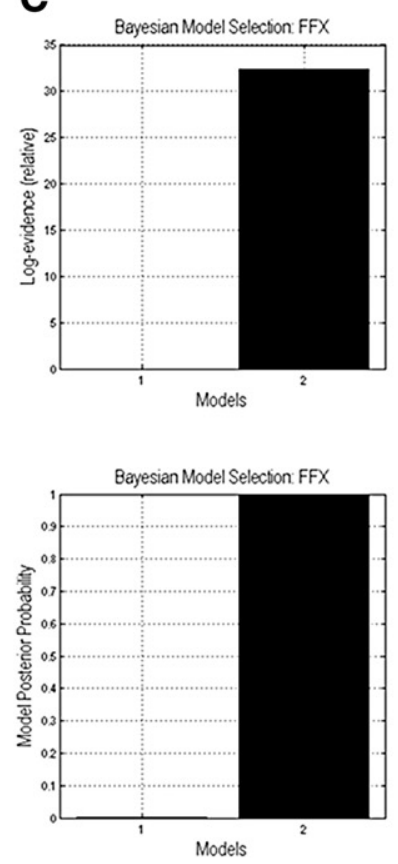

hd-EEG
D
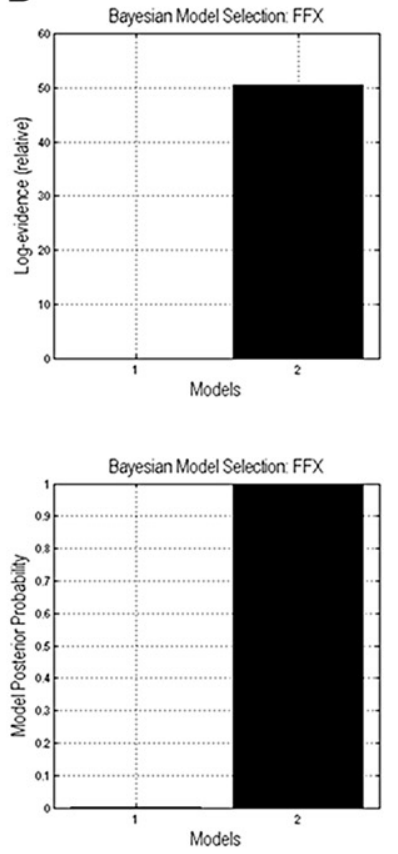

MEG

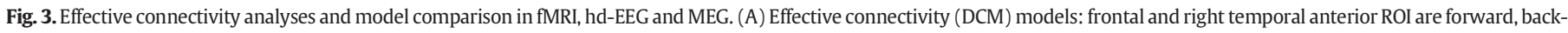

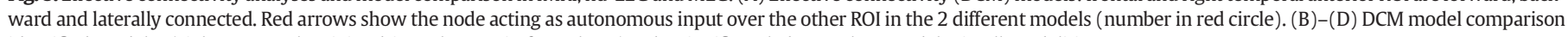
identified model 2 (right temporal activity drives changes in frontal ROI) to be significantly better than model 1 in all modalities. 
A

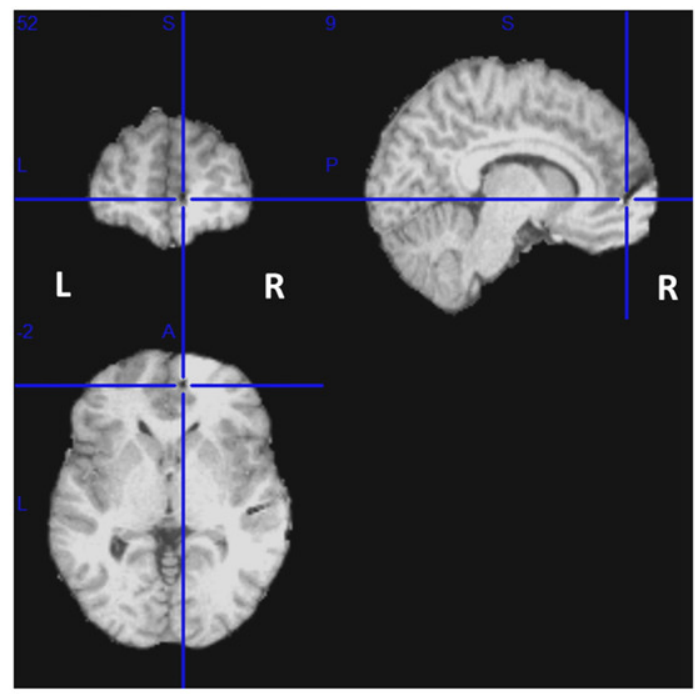

B

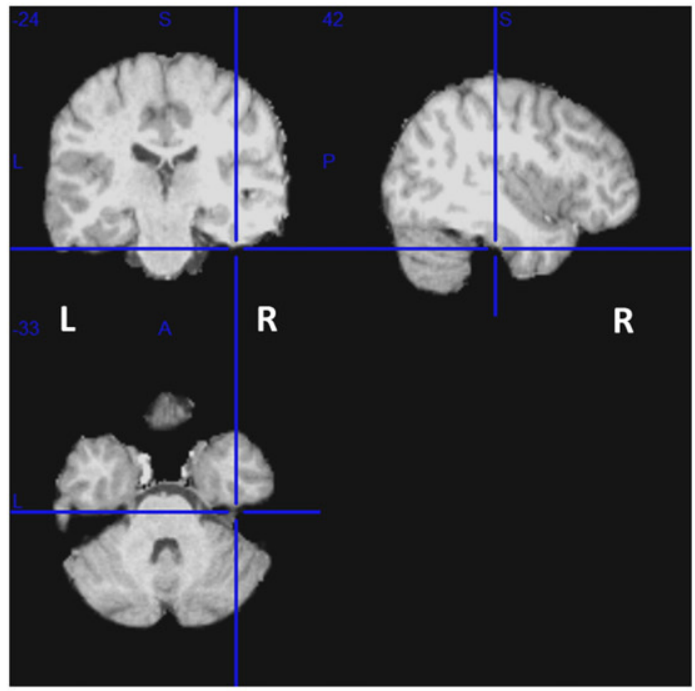

C

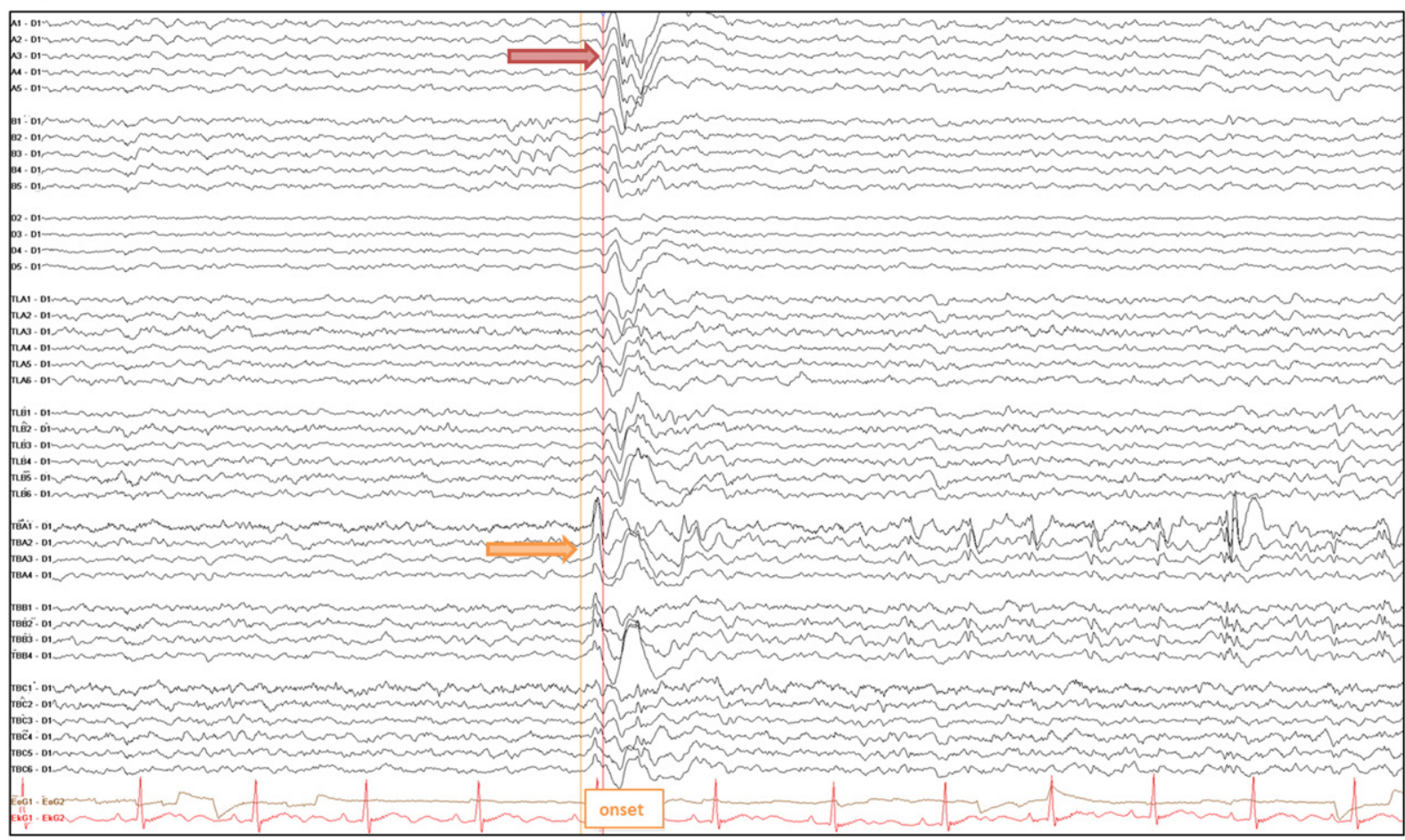

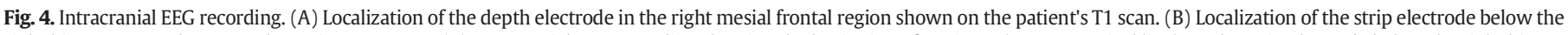

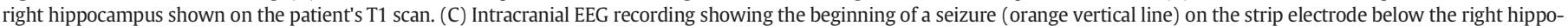
campus (orange arrow) with propagation within $115 \mathrm{~ms}$ to the right mesial frontal depth electrode (red vertical line, red arrow).

epileptic discharges (Marrosu et al., 2009; Morocz et al., 2003; Pittau et al., 2008). The alternative hypothesis suggested by previous papers that activity changes in the orbitofrontal lobes, which are believed to be key structures in processing emotional aspects of music (Morocz et al., 2003), reflect emotional arousal related to the music rather than seizure activity per se does not apply to our data either. The apprehensive feeling associated to the music should then be present throughout the whole block of epileptogenic music and not only appear at the beginning of the seizure as it was the case in our patient. With our methods we were able to classify this activation in the mesial frontal lobes as clear propagation of epileptic activity. The right temporal focus as the "driving force" in our case is in accordance with several reports about patients with musicogenic epilepsy who also underwent invasive EEG monitoring and who became seizure free after resection of the thus identified focus (Tayah et al., 2006; Trevathan et al., 1999). Further, our results are in line with current literature on déjà-vu experiences in epilepsy. Bartolomei et al. (2012) demonstrated that déjà-vu is specifically associated with increased interactions between rhinal cortex, amygdala and hippocampus with the hippocampus playing a pivotal role in these interactions.

Right mesial temporal lobe structures can account for several emotional responses in the processing of sensory stimuli, especially musical ones (Blood and Zatorre, 2001; Blood et al., 1999). While our patient stated that he neither especially liked nor disliked rap music, he indicated that the auras were accompanied by a rather unpleasant feeling, which might find its correlate in the activation of the right mesial temporal lobe during the seizure. 
DCM for fMRI has already been successfully used to investigate effective connectivity in epileptic networks identified with EEG-fMRI on a single-subject patient-specific level (Murta et al., 2012; Vaudano et al., 2012,2013 ) as well as in a small group of patients with idiopathic generalized epilepsy (Vaudano et al., 2009). In these cases, the primary source and propagation pathway of epileptic discharges from fMRI data were determined by testing a set of clinically plausible network connectivity models. These studies demonstrate the feasibility of DCM for the analysis of the temporal dynamics of the spreading of epileptic activity in the brain. However, DCM has been criticized concerning its model selection procedure and the supposedly insufficient validation of an individual winning model (Lohmann et al., 2012). For our analysis we chose a restricted number of models based on a priori knowledge derived from our own source analysis results as well as previous literature. By this means we were able to avoid the problem of a large number of possible models including anatomically implausible ones. With regards to model validation, we made use of different modalities, i.e. fMRI, hd-EEG and MEG, to reaffirm the results of each method. In fact, we were able to corroborate fMRI-DCM results with hd-EEG/MEGDCM, thereby taking advantage not only of the good spatial resolution of fMRI but also of the very high temporal resolution of EEG and MEG. The network models underlying DCM for fMRI on the one hand and DCM for EEG and MEG on the other hand are adapted to each methods particularity, i.e. the slow BOLD response in fMRI and the temporal resolution within the range of milliseconds in EEG and MEG. Differences between DCM for AMRI and for EEG and MEG concern the neuronal model as well as the forward model. In the neuronal model in DCM for fMRI each region is represented by a single hidden state using a bilinear differential equation. Propagation delays are not modeled because fMRI data does not contain enough temporal information due to the large regional variability in hemodynamic response latencies. In DCM for EEG and MEG the neuronal model is more detailed and realistic, describing interactions between different neural subpopulations. Further, it models propagation delays between regions. Regarding the forward model, DCM for fMRI is more complex having to model the transformation of neuronal activity into a BOLD response, whereas DCM for EEG and MEG can use a simple linear model to predict the observed scalp data (Friston et al., 2003; Kiebel et al., 2008). Therefore, the fact that both the fMRI and the EEG and MEG effective connectivity analysis gave very similar results was reassuring in this specific subject but also for DCM in general. We were able to confirm our DCM results with invasive EEG recordings, a means to directly record neuronal activity and therefore the gold standard for the localization of brain activity. This further proves the applicability of the method and the correctness of our results and underlines the usefulness of DCM to investigate epileptic networks and especially to determine focus and propagation of epileptic activity.

As a limitation of this study one might discuss that the invasive EEG recording did not sample activity from "within" the hippocampus, i.e. the winning ROI, but from the basal mesial temporal region right below the hippocampus. However, sampling activity from the mesiotemporal structures with multiple strip electrodes from temporo-basal (with the most distal electrodes close to the hippocampus) is a standard approach for mesio-temporal studies in epilepsy surgery. It has been shown that subdural strip electrodes, if placed correctly as it was the case in our patient, are able to localize the seizure focus as accurately as depth electrodes in the mesial temporal lobe (Eisenschenk et al., 2001).

\section{Conclusions}

In summary, we could show the utility of DCM for the characterization of epileptic networks in a case of musicogenic epilepsy, a unique scenario to trigger epileptic network activity by an external stimulus. Using the complementary nature of fMRI and EEG/MEG in combination with advanced network analysis tools, we could non-invasively unravel the temporal sequence of epileptic activity spread. The techniques applied may be useful to determine the focus and characterize network activity in other epilepsy syndromes as well. However, their general applicability remains to be demonstrated in a larger cohort.

\section{Acknowledgments}

Silke Klamer was supported by the fortüne-Programm (2055-0-1) of the University of Tübingen. The study was further supported by the AKF-Programm (289-0-0) of the University of Tübingen, the Center for Integrative Neurosciences (2011-14) and the Deutsche Forschungsgemeinschaft (2388/36-1).

\section{Disclosure of conflicts of interest}

None of the authors have potential conflicts of interest to be disclosed. Supplementary data to this article can be found online at http://dx. doi.org/10.1016/j.neuroimage.2015.03.027.

\section{References}

Barkley, G.L., Baumgartner, C., 2003. MEG and EEG in epilepsy. J. Clin. Neurophysiol.: Off. Publ. Am. Electroencephalogr. Soc. 20, 163-178.

Bartolomei, F., Barbeau, E.J., Nguyen, T., McGonigal, A., Regis, J., Chauvel, P., Wendling, F., 2012. Rhinal-hippocampal interactions during deja vu. Clin. Neurophysiol.: Off. J. Int. Fed. Clin. Neurophysiol. 123, 489-495.

Blood, A.J., Zatorre, R.J., 2001. Intensely pleasurable responses to music correlate with activity in brain regions implicated in reward and emotion. Proc. Natl. Acad. Sci. U. S. A. 98, 11818-11823.

Blood, A.J., Zatorre, R.J., Bermudez, P., Evans, A.C., 1999. Emotional responses to pleasant and unpleasant music correlate with activity in paralimbic brain regions. Nat. Neurosci. 2, 382-387.

Brodbeck, V., Spinelli, L., Lascano, A.M., Wissmeier, M., Vargas, M.I., Vulliemoz, S., Pollo, C., Schaller, K., Michel, C.M., Seeck, M., 2011. Electroencephalographic source imaging: a prospective study of 152 operated epileptic patients. Brain: J. Neurol. 134 2887-2897.

Eisenschenk, S., Gilmore, R.L., Cibula, J.E., Roper, S.N., 2001. Lateralization of temporal lobe foci: depth versus subdural electrodes. Clin. Neurophysiol.: Off. J. Int. Fed. Clin. Neurophysiol. 112, 836-844.

Friston, K.J., Harrison, L., Penny, W., 2003. Dynamic causal modelling. Neurolmage 19, 1273-1302.

Kelley, W.M., Miezin, F.M., McDermott, K.B., Buckner, R.L., Raichle, M.E., Cohen, N.J. Ollinger, J.M., Akbudak, E., Conturo, T.E., Snyder, A.Z., Petersen, S.E., 1998. Hemispheric specialization in human dorsal frontal cortex and medial temporal lobe for verbal and nonverbal memory encoding. Neuron 20, 927-936.

Kiebel, S.J., Garrido, M.I., Moran, R.J., Friston, K.J., 2008. Dynamic causal modelling for EEC and MEG. Cogn. Neurodyn. 2, 121-136.

Klamer, S., Elshahabi, A., Lerche, H., Braun, C., Erb, M., Scheffler, K., Focke, N.K., 2014. Differences between MEG and high-density EEG source localizations using a distributed source model in comparison to fMRI. Brain Topogr. 28, 87-94.

Lantz, G., Spinelli, L., Seeck, M., de Peralta Menendez, R.G., Sottas, C.C., Michel, C.M. 2003. Propagation of interictal epileptiform activity can lead to erroneous source localizations: a 128-channel EEG mapping study. J. Clin. Neurophysiol.: Off. Publ. Am. Electroencephalogr. Soc. 20, 311-319.

Lohmann, G., Erfurth, K., Muller, K., Turner, R., 2012. Critical comments on dynamic causal modelling. Neurolmage 59, 2322-2329.

Marrosu, F., Barberini, L., Puligheddu, M., Bortolato, M., Mascia, M., Tuveri, A., Muroni, A. Mallarini, G., Avanzini, G., 2009. Combined EEG/fMRI recording in musicogenic epilepsy. Epilepsy Res. 84, 77-81.

Mazziotta, J.C., Toga, A.W., Evans, A., Fox, P., Lancaster, J., 1995. A probabilistic atlas of the human brain: theory and rationale for its development. The International Consortium for Brain Mapping (ICBM). NeuroImage 2, 89-101.

Morocz, I.A., Karni, A., Haut, S., Lantos, G., Liu, G., 2003. fMRI of triggerable aurae in musicogenic epilepsy. Neurology 60, 705-709.

Murta, T., Leal, A., Garrido, M.I., Figueiredo, P., 2012. Dynamic Causal Modelling of epileptic seizure propagation pathways: a combined EEG-fMRI study. NeuroImage 62 1634-1642.

Pataraia, E., Lindinger, G., Deecke, L., Mayer, D., Baumgartner, C., 2005. Combined MEG/ EEG analysis of the interictal spike complex in mesial temporal lobe epilepsy. Neurolmage 24, 607-614.

Pittau, F., Tinuper, P., Bisulli, F., Naldi, I., Cortelli, P., Bisulli, A., Stipa, C., Cevolani, D., Agati, R., Leonardi, M., Baruzzi, A., 2008. Videopolygraphic and functional MRI study of musicogenic epilepsy. A case report and literature review. Epilepsy Behav.: E\&B 13, 685-692.

Smith, M.L., Milner, B., 1981. The role of the right hippocampus in the recall of spatial location. Neuropsychologia 19, 781-793.

Tayah, T.F., Abou-Khalil, B., Gilliam, F.G., Knowlton, R.C., Wushensky, C.A., Gallagher, M.J., 2006. Musicogenic seizures can arise from multiple temporal lobe foci: intracranial EEG analyses of three patients. Epilepsia 47, 1402-1406. 
Trevathan, E., Gewirtz, R.J., Cibula, J.E., Schmitt, F.A., 1999. Musicogenic seizures of right superior temporal gyrus origin precipitated by the theme song from 'The X-Files'. Epilepsia 40, 23-33.

Vaudano, A.E., Laufs, H., Kiebel, S.J., Carmichael, D.W., Hamandi, K., Guye, M., Thornton, R. Rodionov, R., Friston, K.J., Duncan, J.S., Lemieux, L., 2009. Causal hierarchy within the thalamo-cortical network in spike and wave discharges. PLoS ONE 4, e6475.

Vaudano, A.E., Carmichael, D.W., Salek-Haddadi, A., Rampp, S., Stefan, H., Lemieux, L., Koepp, M.J., 2012. Networks involved in seizure initiation. A reading epilepsy case studied with EEG-fMRI and MEG. Neurology 79, 249-253.
Vaudano, A.E., Avanzini, P., Tassi, L., Ruggieri, A., Cantalupo, G., Benuzzi, F., Nichelli, P., Lemieux, L., Meletti, S., 2013. Causality within the epileptic network: an EEG-fMRI study validated by intracranial EEG. Front. Neurol. 4, 185

Vulliemoz, S., Thornton, R., Rodionov, R., Carmichael, D.W., Guye, M., Lhatoo, S., McEvoy, A.W., Spinelli, L., Michel, C.M., Duncan, J.S., Lemieux, L., 2009. The spatio-temporal mapping of epileptic networks: combination of EEG-fMRI and EEG source imaging. NeuroImage 46, 834-843. 